\title{
Inefficiency of equilibria in query auctions with continuous valuations - Extended Abstract
}

\author{
Elena Grigorieva ${ }^{1}$, P. Jean-Jacques Herings ${ }^{2}$, Rudolf Müller ${ }^{1}$, and Dries \\ Vermeulen ${ }^{1}$ \\ 1 Department of Quantitative Economics, Maastricht University, P.O. Box 616, 6200 \\ MD Maastricht, The Netherlands. \\ ${ }^{2}$ Department of Economics, Maastricht University, P.O. Box 616, 6200 MD \\ Maastricht, The Netherlands.
}

\begin{abstract}
Query auctions are (iterative) auctions in which bidders have to select (in each round) from a finite set of actions. We show that, when bidders have continuous valuations, any ex post equilibrium in an ex post individually rational query auction can only be ex post efficient when the running time of the auction is infinite for almost all realizations of valuations of the bidders. In contrast we show that, when we allow for inefficient allocations with arbitrarily small probability, there is a query auction (to be more specific, a bisection auction) that attains this level of approximate efficiency in equilibrium, while additionally the running time of the auction in equilibrium is finite for all realizations of valuations.
\end{abstract}

Keywords and Phrases: Query auctions, ex post equilibrium, efficiency.

JEL Classification Numbers: C72, D44.

\section{Introduction}

This paper concerns the sales of a single indivisible item to $n$ buyers by means of an auction, each buyer having a private valuation $v_{i}$ for the item. ${ }^{3}$ This setting has been thoroughly analyzed and is very well understood (see for example Krishna (2002)). The sealed-bid second price auction by Vickrey (1961), and the ascending clock version of the English auction are two strategically equivalent designs that solve the allocation problem in dominant strategies. In the dominant strategy equilibrium both auctions allocate the item efficiently -they both award the item to the bidder with the highest valuation. The Vickrey auction collects sealed bids, allocates to the bidder with the highest bid (if more than one, using a lottery), and sets the price equal to the second-highest bid. The ascending clock version of the English auction continuously increases the price on a price clock, and bidders step out if the price becomes larger than their willingness to pay. The clock stops when the second last bidder stepped out. If at some point

\footnotetext{
${ }^{3}$ We assume quasi-linear utilities throughout the paper.
} 
all remaining bidders step out simultaneously, the item is assigned by a lottery among them at the current price.

When bidder's valuations are continuous, that is, may take any real number in some interval $[\alpha, \beta]$, practical implementations of both auctions face a problem. The strategic properties of the Vickrey auction rely on exact communication of valuations between bidders and auctioneer. However, whatever communication language we use, the number of expressible elements in $[\alpha, \beta]$ will be countable, while $[\alpha, \beta]$ is uncountable. Therefore no such language is sufficient to communicate the necessary information in the Vickrey auction. The clock version of the English auction requires that bidders step out at exactly their valuation, which disallows any discrete clock increments. The question arises whether there is at all any practically implementable auction in case of continuous valuations that shares the properties of the Vickrey auction, and, if not, to which extend its properties have to be given up in a practical implementation. Informally, a practical implementations should not rely on bids with infinitely long encoding or a continuous clock. We shed light on these questions by providing negative as well as positive results. As part of our analysis we offer a definition of practically implementable auctions and call such auctions query auctions. Query auctions are (iterative) auctions in which bidders have to select (in each round) from a finite set of actions.

We show that for every query auction in equilibrium necessarily the item will be assigned inefficiently with positive probability, unless one accepts the unrealistic phenomenon that the auction will last indefinitely -i.e. the auction runs an infinite number of rounds- for almost all realizations of valuations. In other words, any implementation of the Vickrey auction by means of a query auction in a model with continuous valuations will necessarily have an infinite running time for almost all realizations of valuations, and is hence not a feasible option in any practical sense. On the other hand, we show that the probability of assigning the item inefficiently can be made arbitrary small. More precisely, we define a family of query auctions, parameterized by values $c \in(0,1]$ and called bisection auctions, for which in equilibrium the probability of inefficient allocation is at most $c$.

At first glance, one might argue that these results are of theoretical interest only as the assumption of continuous valuations might by itself not hold in practice. In other words, a theory of implementable auctions should anyhow assume discrete sets of valuations. However, our results for continuous valuations are also of interest in discrete settings. Firstly, assume that the numeric precision of discrete valuations varies among bidders, and is furthermore not known by the auctioneer. This is imaginable in auctions used in distributed, computerized systems, for example for network resource allocation. In such cases our positive results offer the possibility to make the pessimistic assumption that valuations are continuous and then use a bisection auction that performs well even for this setting. Thereby we will need no a-priori agreement on discrete bid levels. Another setting of interest are integer valuations that are very large, say in 
the range of billions of Euro, as in spectrum auctions. In such settings, bid increments will have to be very large if they should lead to a reasonable number of rounds of the English auction (or its combinatorial counterpart of a simultaneous ascending auction (SAA) Milgrom (2000)), giving the set of valuations a flavor of continuity. As a bisection auction performs well even for continuous valuations, they could provide a reasonable alternative to designs with a priori fixed bid increments.

We will now turn to a more detailed description of the exact environment in which and the exact conditions under which our results hold.

Continuous valuations. Throughout the paper we assume that valuations can take any real number in an interval $[\alpha, \beta) \subset \mathbb{R} .{ }^{4}$ When analyzing the proposed bisection auctions, we assume further that the types are identically, independently distributed, with a continuous cumulative density. These assumptions on type distributions are however only of importance for analyzing the probability of inefficient allocation. All game theoretic properties of bisection auctions are independent of any assumption on type distributions. In particular, they hold also for correlated type distributions.

Query auctions. In a query auction the auctioneer sequentially offers bidders the opportunity to take one of a finite set of actions. Such an offer is referred to as a query. ${ }^{5}$ During the course of the auction each bidder may be, and usually will be, queried more than once. Typically an "action" takes the form of an answer to a query regarding the valuation of the bidder in question, such as "Is your valuation larger than 15?" to which the response can be either a yes or a no.

Determination of winner and payment in a query auction are based exclusively on the actions taken by the bidders in response to the queries of the auctioneer. The auction ends as soon as both winner and payment are determined. The number of times a particular bidder is queried during the auction is not assumed to be bounded, and the auction may thus potentially take an infinite number of query rounds. We will only consider ex post individually rational query auctions, meaning that each bidder, given his valuation $v_{i}$, has a plan of

\footnotetext{
${ }^{4}$ Our negative results on query auctions would not change if we weaken this assumption. All what we need is that the elements in $[\alpha, \beta]$ that can occur have a positive Lebesgue measure.

${ }^{5}$ Each separate query by the auctioneer could be thought of as a round in the auction because the action taken by the queried bidder is, at least in our setting, supposed to be publicly observable. Only actions whose effects can only be observed at the same moment in time by other bidders are usually considered to be taken in the same round. Rounds typically differ from each other in terms of the information available to bidders. In that sense each query could be counted as a round. In this paper though we deviate slightly from this standard interpretation. The order in which bidders are queried is usually fixed, and a round is a sequence of queries in which each bidder is queried exactly once.
} 
action in the auction that guarantees him a non-negative payoff, regardless of the behavior of the other bidders.

Inefficiency of ex post equilibrium. In this paper we investigate the efficiency of ex post equilibria in query auctions. An ex post equilibrium is a strategy profile such that, given any realization of valuations, the plan of action prescribed to a bidder in the auction by his strategy is a best response to the plans of action prescribed by the strategies of the other bidders given their valuations. The first main result in this paper can now be precisely formulated as follows. An ex post equilibrium is called sometimes finite if the set of realizations of valuations for which in equilibrium the auction ends in finite time has positive Lebesgue measure. The result is that, given any ex post individually rational query auction, any ex post equilibrium in that auction that is sometimes finite cannot be ex post efficient.

Existence of ex post equilibrium in bisection auctions. The first result poses the question how much we need to give up in efficiency in order to be able to find query auctions that are individually rational and sometimes finite. In order to answer this question we show first that for a very wide class of query auctions, namely the so-called bisection auctions, an individually rational ex post equilibrium exists. Moreover, under a mild assumption (namely that the price in the auction can in principle be driven up to exceed any possible valuation of any bidder, an assumption that is met by all existing auctions) the equilibrium can even be shown to be finite for any realization of valuations.

The bisection mechanism works as follows. Valuations of bidders are assumed to be drawn from an interval $I=[\alpha, \beta)$. Before the auction starts, an order of the bidders in the auction is chosen randomly. We assume that this ordering is $1 \prec 2 \prec 3 \prec \cdots \prec n-1 \prec n$.

The auction runs for an a priori indefinite number of rounds. In each round there is a specific payment $P$ to be made by a bidder if he wins in this round. In every round there is also a query price $Q$ which is higher than the current payment, and an upper bound $H$ on future payments that is higher than the query price. Initially the payment and the upper bound are set as $P=\alpha$ and $H=\beta$, and all bidders are active. In every round the auctioneer asks the bidders that are active in that round whether they would be willing to pay the query price. Bidders are queried openly in increasing order.

If only one bidder is willing to pay the query price, he becomes the winner of the auction. He has to pay the current payment (not the query price). If more than 1 bidder is willing to pay the query price, the auction proceeds into the next round. Only those bidders who agreed to pay the query price stay active. The query price becomes the payment, and the new query price is raised to a level strictly above the old query price, but still below the upper bound. If no bidder is willing to pay the query price all bidders stay active, the payment stays the same, the old query price becomes the new upper bound, and the new query price is set between the payment and the new upper bound. In case no winner is 
found, i.e., should the auction run indefinitely, then among the bidders who are still active the one with highest ranking wins and he pays the lowest price that is still higher than or equal to any of the payments that were announced while the auction was running.

Effectively a bisection auction is a variation of the bisection auction presented in Grigorieva et al. (2007), the main two differences being that in the present paper the auction may last indefinitely, and that the auction stops as soon as the winner is found. The bisection auction in Grigorieva et al. (2007) was designed to handle the situation in which bidders have discrete valuations. The present definition of a bisection auction is specifically designed to handle continuous valuations.

Given a bisection auction we construct a specific ex-post equilibrium, called the bluff equilibrium, for that auction. The bluff equilibrium requires each bidder to act as follows. When there still is an active bidder with a lower rank in the ordering, the bidder stays in the auction until the query price exceeds his valuation. As soon as he becomes the active bidder with the lowest rank, he stays in the auction until the payment exceeds his valuation (effectively a bluff since he will say yes to a query price exceeding his valuation). We show that this strategy is ex post individually rational, and that the resulting profile where each bidder uses this strategy is an ex post equilibrium. We also show that the bluff equilibrium is sometimes finite -and hence not ex post efficient according to our first result. Furthermore, we show that the query price is increasing in the bluff equilibrium.

In the second half of the paper we analyze exactly how (in)efficient the bluff equilibrium may be. In particular we show that approximate efficiency can be achieved within the family of $c$-fraction auctions, a special class of bisection auctions.

$c$-fraction auctions. In a $c$-fraction auction, given the payment $P$ and upper bound $H$ in any round, the query price in that round is given by $Q=(1-c) P+$ $c H$, where $c$ is a rational number in the interval $(0,1)$. Thus, the increment with which the payment $P$ is increased is a fixed fraction $c$ of the current price interval $[P, H)$ (the interval that contains all future payments, no matter what responses the bidders give to future queries).

As a measure of inefficiency we employ the probability of inefficient allocation. We assume that valuations of bidders are drawn independently from the uniform distribution on the interval $[\alpha, \beta) .{ }^{6}$ The probability of inefficient allocation is the probability -according to the joint probability distribution on $[\alpha, \beta)^{n}-$ of the set of realizations of valuations for which in equilibrium the item does not get assigned to a bidder with the highest valuation.

\footnotetext{
${ }^{6}$ We use the uniform distribution merely for ease of exposition. Our results in the second part of the paper hold as soon as valuations are i.i.d. draws from an arbitrary probability distribution on $[\alpha, \beta)$ with a continuous cumulative density.
} 
The main finding in this part of the paper is that for the $c$-fraction auction the probability of inefficient allocation is smaller than or equal to $c$, no matter how many bidders participate in the auction. Moreover, the running time of any $c$ fraction auction in the bluff equilibrium is finite for all realizations of valuations ${ }^{7}$. Thus, the minimum level of efficiency can be determined by the auctioneer before it is known how many bidders will participate in the auction by choosing the appropriate fraction $c$, and finite running time is guaranteed. However, we also show that the probability of inefficient allocation is bounded away from zero. In other words, given the fraction $c$, the probability of inefficient allocation does not converge to zero as the number of participants becomes large. This implies that $c$ is the only tool available to the auctioneer to control the level of inefficiency, increasing the number of participants is not an appropriate method.

As a comment on the full generality of these statements, we stress again that the same conclusions can be obtained for any continuous probability distribution on $[\alpha, \beta)$ from which valuations are independently drawn. In this general setting we construct a variant of the c-fraction auction for which all the above claims hold as well.

These results alleviate the severity of our initial inefficiency result. The inefficiency result said that in our setting efficiency can only be achieved at the expense of an infinite running time of the auction for almost all realizations of valuations. The second part of the paper on the other hand shows that, by choosing the appropriate auction, we can have approximate efficiency (meaning that the probability of inefficient allocation can be made arbitrarily small) in equilibrium, while the running time of the auction in equilibrium is finite for all realizations of valuations. Moreover, given the desired level of efficiency, the particular choice of auction can be made independently of the number of bidders that will participate in the auction.

Related literature. It is common sense in auction design that ascending auctions should utilize minimum bid increments. In case of continuous valuations, one typically requires that new bids are at least some $\epsilon$ higher than standing high bids. Demange et al. (1986) proposed this approach for multi-item auctions with bidders who demand at most one item. Ausubel and Milgrom (2002) use it in ascending combinatorial auctions. The advantage of our bisection auction in comparison to designs with minimum increments is an expected number of rounds that is only logarithmic in the number of bidders, independent of the length of the interval $[\alpha, \beta)$ from which the valuations are drawn (Grigorieva et al. (2006)).

Rothkopf and Harstad (1994) study a model with continuous valuations where bidders are obedient and can only bid on a finite number of bid levels. They analyze how the choice of bid levels influences efficiency and revenue under the assumption of straightforward bidding by the bidders. They derive bounds on the loss of welfare and show that, when valuations are uniformly distributed,

\footnotetext{
${ }^{7}$ In Grigorieva et al. (2006) it is shown that the expected running time is $O(\log n)$.
} 
for 2 bidders and $m$ bid levels the evenly spaced bid level auction is revenue optimal as well as welfare optimal, with an expected loss of welfare of at most $\frac{1}{6 m^{2}}$. In the same model David et al. (2005) argue that a slightly modified version of the auction with discrete bid levels has truthful bidding as a dominant strategy equilibrium. They show that in equilibrium for more than 2 bidders the optimal auction has decreasing bid increments, and subsequently analyze the probability of inefficient allocation, both in the case of evenly spaced bid levels and if bid levels are chosen optimally. In both papers the optimal choice of bid levels depends on the number of bidders, while we derive bounds on the probability of inefficient allocation and the expected loss of welfare that are independent of the number of bidders.

Also Blumrosen and Nisan (2002), Blumrosen et al. (2003), Blumrosen et al. (2007) and Blumrosen and Feldman (2006) study a model where bidders have continuous valuations and a finite set of bid levels. They show that in their model truthful bidding is a dominant strategy equilibrium and derive bounds on the loss of welfare in equilibrium. The loss of welfare for equally spaced $k$ bid levels is $O\left(\frac{1}{k^{2}}\right)$, where $k \geq n$ has to hold. Blumrosen and Feldman (2006) show that the lower bound $n$ for $k$ can be omitted if different bid levels are used for different bidders. But then the $O\left(\frac{1}{k^{2}}\right)$ is a bound for fixed $n$, as $n$ is part of the constant. Again our approach allows to design of auctions that achieve approximate efficiency close to 0 independent of the number of bidders.

Parkes (2005) studies a model in which bidders are uncertain about their preferences and preference elicitation is costly. He shows that in such an environment ascending price query auctions can achieve better allocative efficiency than a sealed bid auction, using less elicitation of preferences.

Notably, our bisection auctions are ascending price auctions, as the payment is increasing or stays the same in any round. However, query prices might be decreasing, and there can be a difference between a query price to which a bidder has committed himself and the price he has to pay. Similar features can be found in the single-item auctions by David et al. (2005) and combinatorial auctions by Mishra and Parkes (2007). Grigorieva and Strobel (2006) show in an experimental evaluation for the case of integer valuations this is of no negative influence on equilibrium bidding.

Organization of the full paper. Section 2 is a preliminary section where we collected most of the known results on auction design that we use in this paper. In section 3 we show that, when valuations are continuous, efficiency of ex post equilibria in query auctions can only be obtained at the expense of an infinite running time of the auction for almost all realizations of valuations. In section 4 we provide a family of query auctions, the bisection auctions, in which we prove the existence of an ex post individually rational ex post equilibrium, called the bluff equilibrium, that has a finite running time for a non-negligible set of valuations. Hence bluff equilibria are ex post inefficient. In section 5 we show that, when we allow for inefficient allocations with arbitrarily small -but positive- probability, there is a bisection auction that attains this level of inefficiency in equilibrium, while the running time is finite for every realization of 
valuations. Section 6 concludes. Appendix 1 is devoted to an elementary proof of the Theorem of Green and Laffont in our (simple) context. Appendix 2 contains proofs of statements that are used in section 4.

The full paper is available as METEOR research memorandum ${ }^{8}$. In a companion paper (Grigorieva et al. (2006)), we offer a more detailed study of the performance of c-bisection auctions.

The first author acknowledges support by the Dutch Science Foundation NWO through grant 401-01-101. The second author acknowledges support by the Dutch Science Foundation NWO through a VICI-grant.

${ }^{8}$ Available at http://edata.ub.unimaas.nl/www-edocs/ 


\section{Bibliography}

Ausubel, L. and P. Milgrom (2002), Ascending Auctions with Package Bidding, in Frontiers of Theoretical Economics, 1: 1-42.

Blumrosen, L. and N. Nisan (2002), Auctions with severely bounded communication, in Proceedings of 43th Annual Symposium on Foundations of Computer Science, Vancouver, Canada, 406-416.

Blumrosen, L., N. Nisan and I. Segal (2003), Multi-player and multi-round auctions with severely bounded communication, in Proceedings of 11th annual European Symposium on Algorithms, Budapest, Hungary.

Blumrosen, L. and M. Feldman (2006), , in Feigenbaum, J., J. Chuang and D. Pennock (eds.), Proceedings of 7th ACM Conference on Electronic Commerce, Ann Arbor, Michigan, Pages 62-71.

Blumrosen, L., N. Nisan and I. Segal (2007), Auctions with severely bounded communication, in Journal of Artificial Intelligence Research, 28: 233-266.

David, E., A. Rogers, J. Schiff, S. Kraus and N.R. Jennings (2005), Optimal design of English auctions with discrete bid levels, in Proceedings of ACM Conference on Electronic Commerce, Vancouver, Canada, 98-107.

Demange, G., D. Gale, and M. Sotomayor (1986), Multi-item auctions, in Journal of Political Economy, 94: 863-872.

Grigorieva E., P.J.J. Herings, R. Müller and D. Vermeulen (2006), The family of c-bisection auctions: efficiency and running time, METEOR research memorandum RM06019.

Grigorieva E., P.J.J. Herings, R. Müller and D. Vermeulen (2007), The private value single item bisection auction, in Economic Theory, 30: 107-118.

Grigorieva E. and M. Strobel (2006), Bidding behavior in the bisection auctionan-an experimental investigation, METEOR research memorandum RM06026.

Green J., and J.J. Laffont (1977), Characterization of satisfactory mechanisms for the revelation of preferences for public goods, in Econometrica, 45: 427-435.

Krishna, V. (2002), Auction Theory, Academic Press, Elsevier Publishers, USA.

Milgrom, P. (2000), Putting auction theory to work-the Simultaneous Ascending Auction, in Journal of Political Economy, 108: 245-272.

Mishra D., and D. V. Parkes (2007), Ascending Price Vickrey Auctions for General Valuations, in Journal of Economic Theory, 132: 335-366.

Parkes, D. (2005), Auction design with costly preference elicitation, in Annals of Mathematics and AI, Special issue on the foundations of electronic commerce, 269-302.

Rothkopf, M.H. and R.M. Harstad (1994), On the role of discrete bid levels in oral auctions, in European Journal of Operational Research, 74: 572-581.

Vickrey W. (1961), Counterspeculation, auctions and competitive sealed tenders, in Journal of Finance 16, 8-37. 